\title{
Non-Unitarity vs Sterile neutrino searches
}

\author{
Josu Hernandez-Garcia* \\ SISSA/INFN - Sezione di Trieste \\ E-mail: josu.hernandezats.infn.it
}

\begin{abstract}
Neutrino masses are one of the most promising open windows to physics beyond the Standard Model (SM). Several extensions of the SM which accommodate neutrino masses require the addition of right-handed neutrinos to its particle content. These extra fermions will either be kinematically accessible (sterile neutrinos) or not (deviations from Unitarity of the PMNS matrix) but at some point they will impact neutrino oscillation searches. We explore the differences and similitudes between the two cases and compare their present bounds with the expected sensitivities of DUNE. We conclude that Non-Unitarity (NU) effects are too constrained to impact present or near future neutrino oscillation facilities but that sterile neutrinos can play an important role at long baseline experiments.
\end{abstract}

Neutrino Oscillation Workshop (NOW2018)

9 - 16 September, 2018

Rosa Marina (Ostuni, Brindisi, Italy)

\footnotetext{
* Speaker.

${ }^{\dagger}$ This project has received funding/support from the European Union's Horizon 2020 research and innovation programme under the Marie Skłodowska-Curie grant agreement No 674896.
} 


\section{Introduction}

The simplest extension of the Standard Model (SM) of particle physics able to account for the evidence for neutrino masses and mixings consists in the addition of right- handed neutrinos to its particle content. The new physics scale is the Majorana mass of the new states and, since it is not related to the electroweak symmetry breaking mechanism, there is no theoretical guidance for its value. A large Majorana scale leads to the celebrated seesaw mechanism [1, 2, 3, 4], providing a very natural explanation of the lightness of neutrino masses. Conversely, a light neutrino mass could also naturally stem from a symmetry argument $[5,6,7,8,9,10]$. This proceeding is based on [11] where we analyze the phenomenological impact of these new physics in neutrino oscillation facilities. If the new mass scale is kinematically accessible in meson decays, the sterile states will be produced in the neutrino beam. On the other hand, if the extra neutrinos are too heavy to be produced, the effective $3 \times 3$ PMNS matrix will show unitarity deviations. We will refer to these situations as sterile and Non-Unitary (NU) neutrino oscillations, respectively. The aim of our work is to discuss the similitudes and differences among these two regimes clarifying in which limit they lead to the same neutrino oscillation phenomenology.

\section{Non-Unitarity vs Sterile neutrinos oscillations}

If $\mathrm{n}$ extra right-handed neutrinos are added to the SM Lagrangian, the full Unitary mixing matrix $U$ can be written as

$$
U=\left(\begin{array}{cc}
N & \Theta \\
R & S
\end{array}\right),
$$

where $N$ represents the $3 \times 3$ active-light sub-block (i.e., the PMNS matrix), which will no longer be unitary, and where $\Theta$ is the $3 \times n$ sub-block that includes the mixing between active and heavy states. Notice that the rotation among sterile and light (heavy) states $R(S)$ is not involved when considering oscillations among active flavors.

The deviations of Unitarity in the leptonic mixing matrix that enters in the CC interactions are encoded in the lower triangular matrix $\alpha[12,13,14,11]$

$$
N=(I-\alpha) U_{\mathrm{PMNS}},
$$

where $\alpha$ is related with the mixing square of the new states.

\subsection{Non-Unitarity: very heavy neutrinos $\left(m>\Lambda_{\mathrm{EW}}\right)$}

In the case of NU, only the light states are kinematically accessible, and therefore the neutrino oscillation probability involves just the $N$ sub-block of Eq. (2.1)

$$
P_{\alpha \beta}=\sum_{i, j} N_{\alpha i} N_{\beta i}^{*} N_{\alpha j}^{*} N_{\beta j} e^{-i \frac{\Delta m_{i j}^{2} L}{2 E}} .
$$




\subsection{Sterile neutrinos: very light masses $\left(m<m_{\pi}\right)$}

In the case of light sterile neutrinos, the new states are produced in the experiment and they participate in the oscillation. Thus, the neutrino oscillation probability will depend on $N$ and on $\Theta$

$$
\begin{aligned}
P_{\alpha \beta} & =\sum_{i, j} N_{\alpha i} N_{\beta i}^{*} N_{\alpha j}^{*} N_{\beta j} e^{-i \frac{\Delta m_{i j}^{2} L}{2 E}}+\sum_{I, J} \Theta_{\alpha I} \Theta_{\beta I}^{*} \Theta_{\alpha J}^{*} \Theta_{\beta J} e^{-i \frac{\Delta m_{I J}^{2} L}{2 E}}+\sum_{I, J} N_{\alpha i} N_{\beta i}^{*} \Theta_{\alpha J}^{*} \Theta_{\beta J} e^{-i \frac{\Delta m_{i j}^{2} L}{2 E}} \\
& \simeq \sum_{i, j} N_{\alpha i} N_{\beta i}^{*} N_{\alpha j}^{*} N_{\beta j} e^{-i \frac{\Delta m_{i j}^{2} L}{2 E}}
\end{aligned}
$$

And therefore, at leading order in $\alpha$, and in the averaged out regime $\left(\Delta m_{i J}^{2} L / 2 E \gg 1\right)$, both limits share the same phenomenology. However, the limits on the $\alpha$ parameters will be different.

\section{Present constraints on Non-Unitarity}

PMNS NU from very heavy extra neutrinos modifies precision electroweak and flavor observables (see for instance $[15,16,17,18]$ ). These modifications translate into very strong upper limits on the $\alpha$ parameters $\mathscr{O}\left(10^{-3}-10^{-4}\right)$ [18]. These deviations of the PMNS matrix are too small to be testes in present and near-future neutrino oscillation experiments, and therefore this case will not further considered in the discussion. However, for sterile neutrinos with masses below the electroweak scale these stringent constraints are lost since the Unitarity is restored. The present constraints on the $\alpha$ parameters for averaged-out regime are $\mathscr{O}\left(10^{-1}-10^{-2}\right)[19,20,21,22,23]$. These deviations from the standard neutrino oscillation probability could be tested in the next generation of neutrino oscillation experiments such as DUNE.

\section{DUNE sensitivities}

The choice of the facility under study is motivated by the strong matter effects that characterize the DUNE setup and that allow to probe not only the source and detector effects induced by the new physics in a given channel $P_{\alpha \beta}$, but also the matter effects which now provide sensitivity to other $\alpha$ parameters. In experiments such as DUNE, the flux and the cross section at the far detector (FD) is normalized with the information of the near detector (ND). Thus, two cases can be studied

- ND averaged case: when the oscillations are averaged out at the ND and FD. The probability would be given by the ratio of Eq. (2.4) at the FD $(L=1300 \mathrm{~km})$ and Eq. (2.4) at the ND $(L=0)$. For the DUNE setup, this scenario is happening for $\Delta m^{2} \gtrsim 100 \mathrm{eV}^{2}$.

- ND undeveloped case: when the oscillations are not developed at the ND yet. The probability would be given by Eq. (2.4) at the FD $(L=1300 \mathrm{~km})$ since the one at the ND would be equal to one. For DUNE, this would be the case only in the region $0.1 \mathrm{eV}^{2} \lesssim \Delta m^{2} \lesssim 1 \mathrm{eV}^{2}$.

Figure 1 show the resulting frequentist allowed regions are shown at $1 \sigma, 90 \% \mathrm{CL}$ and $2 \sigma$. The solid lines represent the expected sensitivities for DUNE, while the dashed lines correspond to the expected sensitivities when using as prior the information of present experiments. The sensitivities to the diagonal parameters $\alpha_{e e}$ and $\alpha_{\mu \mu}$ are significantly stronger for the ND undeveloped (right 

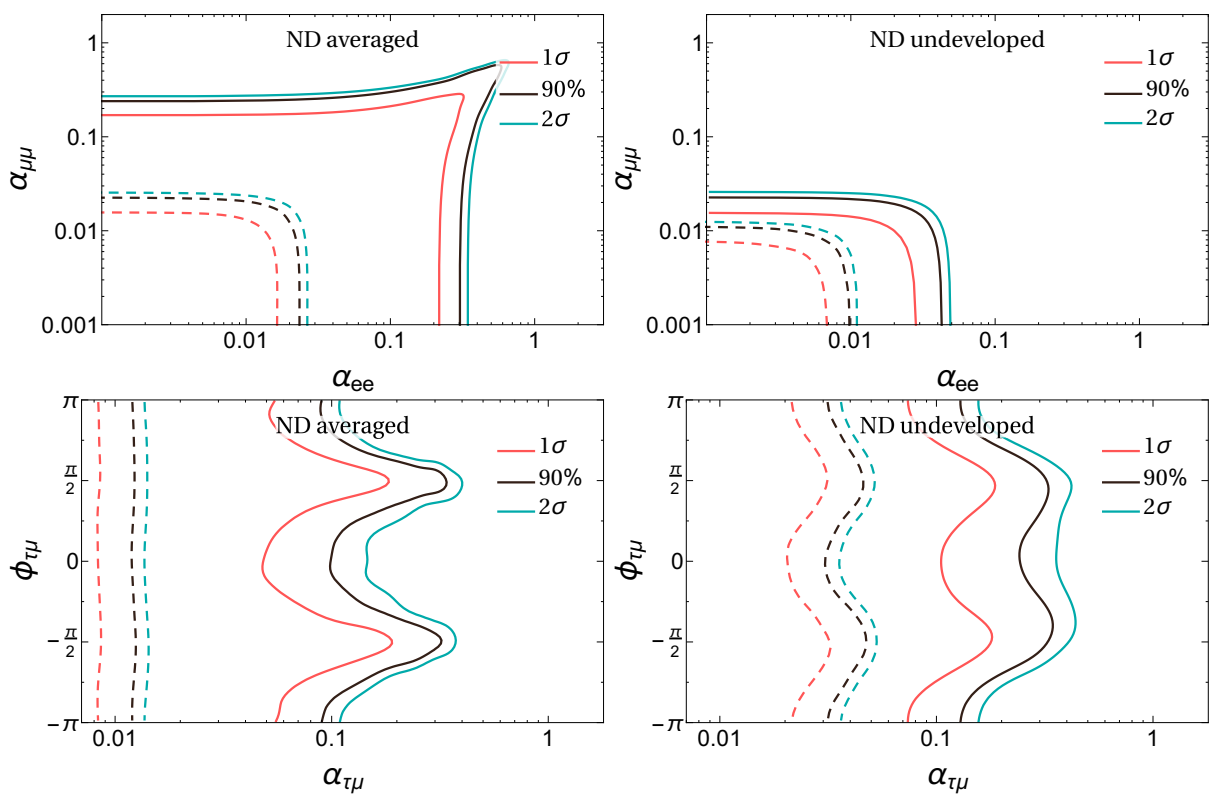

Figure 1: Expected frequentist allowed regions at $1 \sigma, 90 \% \mathrm{CL}$ and $2 \sigma$ for DUNE. The sensitivity to the $\alpha$ parameters not shown here can be found in [11].

panel) as compared to the ND averaged scenario (left panel). This was to be expected since the source and detection effects that provide a leading order sensitivity to the diagonal parameters are totally or partially cancelled once the normalization of the ND is included. In summary, if both ND and FD are affected by the new physics in the same way (as it is the case when the sterile neutrino oscillations are averaged out at both detectors, or in the NU scenario) their effects are more difficult to observe since they cannot be disentangled from the flux and cross section determination at the ND.

\section{Conclusions}

We have shown that, when the sterile neutrino oscillations are averaged out (and at leading order in the small heavy-active mixing angles) both kinematically accessible sterile neutrinos and PMNS NU stemming from heavy new physics lead to the same modifications in the neutrino oscillation probabilities. However, the present constraints which apply to these two scenarios are very different. Indeed, PMNS NU is bounded at the per mille level, or even better for some elements, through precision electroweak and flavor observables, while sterile neutrino mixing in the averaged-out regime is allowed at the percent level since it can only be probed via oscillation experiments themselves. Thus, no impact in present or near-future oscillation facilities from PMNS NU is expected while sterile neutrino mixing could potentially be discovered by them if the sterile neutrinos are light enough to be produced at the source. Indeed, our simulations confirm that PMNS NU is beyond the reach of high precision experiments such as DUNE, but that sterile neutrino oscillations could manifest in several possible interesting ways. Through these simulations the importance of correctly accounting for the impact of the ND was made evident. 


\section{References}

[1] P. Minkowski, $\mu \rightarrow$ e $\gamma$ at a Rate of One Out of $10^{9}$ Muon Decays?, Phys. Lett. 67B (1977) 421.

[2] R. N. Mohapatra and G. Senjanovic, Neutrino Mass and Spontaneous Parity Nonconservation, Phys. Rev. Lett. 44 (1980) 912.

[3] T. Yanagida, HORIZONTAL SYMMETRY AND MASSES OF NEUTRINOS, Conf. Proc. C7902131 (1979) 95.

[4] M. Gell-Mann, P. Ramond and R. Slansky, Complex Spinors and Unified Theories, Conf. Proc. C790927 (1979) 315 [1306.4669].

[5] R. N. Mohapatra and J. W. F. Valle, Neutrino Mass and Baryon Number Nonconservation in Superstring Models, Phys. Rev. D34 (1986) 1642.

[6] J. Bernabeu, A. Santamaria, J. Vidal, A. Mendez and J. W. F. Valle, Lepton Flavor Nonconservation at High-Energies in a Superstring Inspired Standard Model, Phys. Lett. B187 (1987) 303.

[7] G. C. Branco, W. Grimus and L. Lavoura, The Seesaw Mechanism in the Presence of a Conserved Lepton Number, Nucl. Phys. B312 (1989) 492.

[8] W. Buchmuller and D. Wyler, Dilatons and majorana neutrinos, Phys. Lett. B249 (1990) 458.

[9] A. Pilaftsis, Radiatively induced neutrino masses and large Higgs neutrino couplings in the standard model with Majorana fields, Z. Phys. C55 (1992) 275 [hep-ph/ 9901206 ].

[10] J. Kersten and A. Yu. Smirnov, Right-Handed Neutrinos at CERN LHC and the Mechanism of Neutrino Mass Generation, Phys. Rev. D76 (2007) 073005 [0705.3221].

[11] M. Blennow, P. Coloma, E. Fernandez-Martinez, J. Hernandez-Garcia and J. Lopez-Pavon, Non-Unitarity, sterile neutrinos, and Non-Standard neutrino Interactions, JHEP 04 (2017) 153 [1609.08637].

[12] Z.-z. Xing, Correlation between the Charged Current Interactions of Light and Heavy Majorana Neutrinos, Phys. Lett. B660 (2008) 515 [0709.2220].

[13] Z.-z. Xing, Full parametrization of the6 $\tilde{A} U$ Uflavor mixing matrix in the presence of three light or heavy sterile neutrinos, Physical Review D 85 (2012).

[14] F. J. Escrihuela, D. V. Forero, O. G. Miranda, M. Tortola and J. W. F. Valle, On the description of nonunitary neutrino mixing, Phys. Rev. D92 (2015) 053009 [1503.08879].

[15] S. Antusch, C. Biggio, E. Fernandez-Martinez, M. B. Gavela and J. Lopez-Pavon, Unitarity of the Leptonic Mixing Matrix, JHEP 10 (2006) 084 [hep-ph / 0607020 ].

[16] S. Antusch and O. Fischer, Non-unitarity of the leptonic mixing matrix: Present bounds and future sensitivities, JHEP 10 (2014) 094 [1407.6607].

[17] E. Fernandez-Martinez, J. Hernandez-Garcia, J. Lopez-Pavon and M. Lucente, Loop level constraints on Seesaw neutrino mixing, JHEP 10 (2015) 130 [1508 . 03051].

[18] E. Fernandez-Martinez, J. Hernandez-Garcia and J. Lopez-Pavon, Global constraints on heavy neutrino mixing, JHEP 08 (2016) 033 [1605 . 08774].

[19] Y. Declais et al., Search for neutrino oscillations at 15-meters, 40-meters, and 95-meters from a nuclear power reactor at Bugey, Nucl. Phys. B434 (1995) 503.

[20] SUPER-KAMIOKANDE collaboration, K. Abe et al., Limits on sterile neutrino mixing using atmospheric neutrinos in Super-Kamiokande, Phys. Rev. D91 (2015) 052019 [1410 . 20 08 ]. 
[21] MINOS collaboration, P. Adamson et al., Search for Sterile Neutrinos Mixing with Muon Neutrinos in MINOS, Phys. Rev. Lett. 117 (2016) 151803 [1607.01176].

[22] NOMAD collaboration, P. Astier et al., Final NOMAD results on muon-neutrino $\rightarrow$ tau-neutrino and electron-neutrino $\rightarrow$ tau-neutrino oscillations including a new search for tau-neutrino appearance using hadronic tau decays, Nucl. Phys. B611 (2001) 3 [hep-ex/ 0106102 ].

[23] NOMAD collaboration, P. Astier et al., Search for $n u(m u) \rightarrow n u(e)$ oscillations in the NOMAD experiment, Phys. Lett. B570 (2003) 19 [hep-ex/ 0306037 ]. 